Daimon. Revista Internacional de Filosofía, no 82 , 2021 pp. 189-195

ISSN: 1130-0507 (papel) y 1989-4651 (electrónico)

http://dx.doi.org/10.6018/daimon.436061

\title{
Sobre el respeto a la evidencia empírica. McIntyre en La actitud científica
}

\author{
On upholding empirical evidence. \\ McIntyre in The Scientific Attitude.
}

MARIANO SANJUÁN $N^{* *}$

\begin{abstract}
Resumen: No hace mucho tiempo, la demarcación, el cambio teórico o la racionalidad científica coloreaban la paleta de la filosofía de la ciencia. Hoy estos problemas son vistos como asuntos clásicos de la disciplina. En La actitud científica, Lee McIntyre renueva el escaparate filosófico recuperando el problema de la demarcación, defendiendo que lo distintivo de la ciencia es "que se preocupa por la evidencia y está dispuesta a modificar sus teorías en función de la evidencia" $(24)^{1}$. Se presentan a continuación una síntesis de la obra y cinco objeciones.

Palabras clave: Actitud Científica; Evidencia Empírica; Demarcación; Pseudociencia; Negacionismo; Posverdad.
\end{abstract}

\begin{abstract}
Not long ago, demarcation, theory change and scientific rationality used to clutter up debates on the philosophy of science. These issues are now seen as the classical subjects of the discipline. In The Scientific Attitude, Lee McIntyre revamps the philosophical showcase by taking over the demarcation problem by asserting that "what is distinctive about science is that it cares about evidence and is willing to change its theories on the basis of evidence". I hereby synthetize his proposal and offer five objections. Keywords: Scientific Attitude; Empirical Evidence; Demarcation; Pseudoscience; Negationism; Post-truth.
\end{abstract}

La actitud científica [2020 (2019), trad. de Rodrigo Neira, Madrid: Cátedra, 317 pp.] es un intento de reivindicar los logros epistémicos de la ciencia frente a otras formas de conocimiento. A juicio del autor, estos logros son debidos a un tipo de actitud especial respecto a la evidencia empírica que es especialmente característica de la ciencia, aunque no exclusiva. Lo que identifica a la ciencia y la distingue de la pseudociencia, desde este enfoque, es un

Recibido: 07/07/2020. Aceptado: 12/08/2020.

* Agradezco los valiosos comentarios de Paula Olmos a versiones previas. Este trabajo ha sido financiado por la Agencia Estatal de Investigación a través del proyecto "Prácticas argumentativas y pragmática de las razones" (PGC2018-095941B-100).

** Ayudante en el Departamento de Lingüística, Lenguas Modernas, Lógica y Filosofía de la Ciencia, Teoría de la Literatura y Literatura comparada de la Facultad de Filosofía y Letras de la Universidad Autónoma de Madrid. Contacto: mariano.sanjuan@uam.es. Líneas de Investigación: Unidad de la ciencia, Realismo científico, Explicación científica. Publicaciones recientes más relevantes: Sanjuán, M. (2019), "El a priori relativo. ¿Hacia una noción pragmatista de la ciencia?”, ArtefaCToS 8 (2), pp. 139-160. Sanjuán, M. (2018), “Ciencia, idealización y predicción. Los tipos ideales en Max Weber y los conceptos ideales en ciencias de la naturaleza", Thémata 58, pp. 87-106.

1 Todas las referencias a páginas corresponden al libro de McIntyre. 
tipo de actitud respecto a la evidencia. Así, la ciencia "legítima" sería aquella que explícita y conscientemente deja a un lado las creencias e ideologías previas y se pone al servicio de los datos de la experiencia netamente considerados; mientras que la ciencia "ilegítima" sería aquella que permite que los prejuicios y las presuposiciones actúen como filtro a la hora de evaluar la evidencia disponible. Lo que pretende McIntyre en esta obra, en la que resuena el eco de Karl Popper por los cuatro costados, es rastrear el tipo de valores y conductas que identifican el ethos de la buena ciencia.

La actitud científica está dividido en diez capítulos. Los seis primeros están destinados a lo que el autor denomina entender la ciencia; los dos siguientes, a defenderla; y los dos últimos, a expandirla. Como puede entreverse por la estructura narrativa de la obra -entender, luego defender, luego expandir-, la intención de McIntyre es "no solo defender la ciencia, sino hacer proselitismo" (114) de la importancia de sus logros epistémicos ante el auge de propuestas como el diseño inteligente, el terraplanismo, la anti-vacunación o el negacionismo del cambio climático, todas ellas teorías y consignas pseudocientíficas en el mejor de los casos. Frente a ellas, McIntyre sostiene que han de ser defendidas y expandidas el tipo de explicaciones que más robustamente se apoyen en la evidencia disponible. La expansión, que en la anterior frase tiene un sentido social -expandir la ciencia significa ahí convencer de su pertinencia a quien la pone en suspenso-, posee a su vez un sentido disciplinar. El autor sostiene que "si ámbitos como las ciencias sociales desean verse más rigurosos, [...] deben abrazar la actitud científica" (297). Más adelante diré algo sobre este naturalismo de apariencia comteana, sobre su interpretación y la manera en que contrasta con la tendencia popular de la filosofía de la ciencia actual. Por ahora y a continuación, expondré las ideas principales de cada capítulo, ofreciendo asimismo alguna crítica cuando se considere oportuno. Tras ello, lanzaré cinco objeciones a la presente obra.

Es preciso reconocer que el olor a naftalina que desprende la introducción no hace justicia a la frescura intelectual de los subsiguientes capítulos de la obra. Durante las primeras páginas del libro, McIntyre fundamenta su investigación en la premisa de que "casi ningún filósofo de la ciencia afirma hoy que no existe tal cosa como el método científico, que el intento de elaborar un criterio de demarcación está desfasado y que el cientificismo es peligroso" (18), o en la declaración de que el enfoque adoptado en la obra "es muy diferente al de mis predecesores" (23). Es sencillamente complicado estar de acuerdo con estas afirmaciones. No son pocos los autores y autoras que sí han defendido tesis semejantes: Paul Feyerabend (1975), Helen Longino (1990) o el recientemente fallecido Ronald Giere (1999) son solo los primeros nombres que despiertan a la memoria; y sin embargo, estoy convencido de que todos ellos aceptarían de buen grado muchas de las ideas que contiene La actitud científica. Olvidar su producción para justificar la actualidad de la obra resulta artificial y termina por ocultar una buena virtud del libro: que La actitud científica no destaca ni por la novedad de los temas que aparecen en ella ni por la originalidad de la posición defendida, sino por la claridad, transparencia y cuidado que irradia McIntyre a la hora de lidiar con cada uno de ellos. Hay que tener en cuenta que quienes lleguen a este libro estarán, muy probablemente, de acuerdo con las ideas generales que defiende el autor: que la ciencia es racional, que la evidencia desempeña un papel de primer orden en la confirmación de hipótesis y teorías y que el conocimiento científico es preferible a otro tipo de conocimientos por el rigor que requiere su justificación. 
Si por algo es novedosa la posición de McIntyre, es por señalar reiteradamente que la demarcación es una cuestión de respeto a la evidencia y no de método. De esta tesis se ocupa en los dos primeros capítulos de la obra. En el primero de ellos, el autor revisa el problema de la demarcación desde el criterio falsacionista de Karl Popper, cuestionándose, con Larry Laudan (1983), si es posible ofrecer una lista de requisitos necesarios y suficientes exclusivos de la ciencia. McIntrye es perspicaz y su discurso consigue que el lector transite la senda que él mismo pavimenta, argumentando que a pesar de las desavenencias entre las propuestas de Popper y sus críticos -eminentemente Thomas Kuhn-, todos ellos coinciden en que la evidencia es un "sello distintivo de la ciencia" (42). En este mismo capítulo, el autor sopesa y rechaza algunas alternativas contemporáneas a la respuesta popperiana, afirmando que el problema de la demarcación debe consistir en "encontrar una manera de pronunciarse en torno a lo distintivo de la ciencia sin tropezar con el problema de proporcionar unas condiciones necesarias y suficientes" (53).

En el segundo capítulo, McIntyre critica la idea de que la ciencia persigue la verdad. Comienza analizando la tensión entre falsación y corroboración de la que adolece la filosofía popperiana -¿cuántas evidencias refutatorias son necesarias para abandonar una teoría? ¿cuántas confirmaciones hasta que la teoría demuestra su credibilidad? -, para concluir que "la ciencia no puede probar la verdad de ninguna teoría empírica" (62). A pesar de su carácter falible, tenemos buenos motivos para confiar en la ciencia. Nunca sabremos si nuestras mejores teorías son verdaderas, pero sí podemos evaluar si merecen nuestra credibilidad ponderando su consistencia con el conjunto de evidencia disponible en un momento dado. Este razonamiento, que McIntyre denomina la "vindicación pragmática de la creencia fundamentada" (77) es genuinamente -aunque no exclusivamente- científico y constituye el núcleo de la mentalidad o actitud científica.

Los capítulos tercero a sexto dialogan con el concepto de actitud científica. En el tercer capítulo, quizá el más importante del libro, se proporciona una definición al mentado concepto. McIntyre define la actitud científica como el compromiso "honesto y crítico" con dos principios: la preocupación por la evidencia y la disposición al falibilismo. El autor insiste aquí - una vez más- en que esta actitud no tiene nada que ver con la aplicación de un método concreto. Esto es cierto, si por método entendemos un tipo de reconstrucción formal del proceso de investigación à la Wiener Kreis. Pero la sutileza de la matización se diluye si utilizamos el concepto de método de forma más abierta. Un método puede consistir en el compromiso a aplicar una serie de convenciones bien establecidas, como pueden ser la preocupación por la evidencia y la disposición al falibilismo. Si éstos son principios metodológicos en algún sentido y McIntyre cae así en su propia trampa, es algo que debe juzgar el lector.

Surge aquí la siguiente cuestión: ¿cómo va a solventar el problema de la demarcación una definición de ciencia que no es exclusiva de la ciencia? Muy acertadamente, en el cuarto capítulo el autor reconoce que "uno no necesita probar que algo con actitud científica sea ciencia; uno solo necesita mostrar que algo sin actitud científica no es ciencia" (105). Esta maniobra wittgensteiniana de devolver la carga de la prueba al escéptico funciona a las mil maravillas en el caso de la demarcación. Dada la imposibilidad histórica de proporcionar una definición distintiva sobre qué es la ciencia, quizá nuestra mejor baza pase por delimitar qué no lo es. Es preciso apuntar que el autor anticipa en este momento una posible crítica, cuando 
muy oportunamente distingue entre dominios acientíficos y pseudocientíficos. Entre los primeros encontraríamos al arte, la literatura o la filosofía, las cuales no tienen "pretensiones científicas", mientras que el segundo grupo aglomera aquellos discursos que "desean tener protagonismo en la arena empírica, a pesar de desobedecer el estándar de la buena evidencia" (113). McIntyre entiende que solo los segundos cometen impostura. Aunque la distinción es controvertible e imprecisa, el autor hace buen uso de ella para justificar su posición.

En el quinto capítulo, McIntyre argumenta que la actitud científica es un asunto de grupos más que de individuos: "no es solo la honestidad o la buena fe del científico individual, sino la fidelidad a la actitud científica como práctica comunitaria lo que hace a la ciencia especial como institución" (140). Para ello analiza las buenas prácticas -el método estadístico, el peer review y la puesta en común de los datos- y las malas prácticas -errores deliberados, procedimientos negligentes y errores involuntarios- de la ciencia en tanto institución social a través de algunos casos concretos. La tesis del capítulo es doble: por un lado, se defiende que la actitud científica es capaz de mitigar el error con independencia de si su fuente es intencionada -fraude- o involuntaria -sesgos y negligencias. Por otro, se propone que el carácter comunitario de la ciencia no pervierte su objetividad, sino todo lo contrario. La objetividad científica está sostenida sobre un conjunto de estándares compartidos que suscriben todos los miembros de la comunidad, entre los que destaca el respeto a la evidencia. El sexto capítulo ejemplifica a través de una concisa historia de la medicina la transición de la medicina galeana a la práctica clínica moderna, transición catalizada por la adopción comunitaria de la actitud científica.

Como bien arguye McIntyre en los capítulos séptimo y octavo, no todas las historias de la ciencia replican cantos de victoria. En el primero de esta dupla de capítulos, el autor analiza el caso del fraude; en el segundo, el de la ideología ${ }^{2}$. La diferencia entre ambos estriba en las intenciones de quien acomete alguno de ellos. Así, mientras que el fraudulento es plenamente consciente de sus actos, el que actúa movido por creencias ideológicas no tiene la sensación de estar transgrediendo ningún principio epistémicamente valioso. Dicho brevemente: el fraude es a la mentira lo que la ideología a la posverdad. El fraude, que define el autor como la "fabricación o falsificación intencionada de evidencia, encaminada a convencer a alguien para que crea lo que queremos que crea" (210), es el caso más pernicioso de oposición con la actitud científica. El fraude es un acto deliberado que atenta contra los valores que constituyen y sustentan la comunidad científica. A pesar de la gravedad del fraude, McIntyre ilumina cómo los casos más acuciantes suelen salir a la superficie con el tiempo y cómo la comunidad acaba imponiendo graves puniciones a quienes incurren en él. Sin embargo, un caso no tan evidente de mala ciencia es el que proponen los negacionistas y pseudocientíficos. El negacionismo consiste en "el rechazo a adoptar creencias científicas bien fundamentadas" y la pseudociencia consiste en "envolverse del manto de la ciencia para promover una teoría alternativa en torno a una cuestión empírica", rechazando cambiar las creencias incluso a la luz de la evidencia (224-225). En estas situaciones, lo que guía la actuación son intereses económicos, políticos, religiosos o sociales los que llevan a rechazar la evidencia disponible. Así, mientras el fraude pretende parasitar el entramado epistémico de la ciencia, el negacionista directamente considera que tal entramado no posee ningún

2 Aquí McIntyre recopila lo expuesto en su anterior libro; véase McIntyre (2018). 
valor. El capítulo octavo es, junto al tercero, una sección primorosa del libro, pues en él se despliegan las tesis que el lector ya ha asimilado y se ponen a trabajar convincentemente contra el creacionismo, el negacionismo del cambio climático o la parapsicología.

Un detalle importante y original del capítulo octavo es la estrategia que utiliza el autor para rechazar el escepticismo como justificación del negacionismo. Es frecuente encontrar que quienes rechazan las ideas fundamentales del cambio climático lo hacen por motivos escépticos: o bien desconfían del consenso acerca de tales cuestiones, o bien dudan de la precisión de las predicciones menos optimistas. Esto, consideran, es suficiente legitimación como para poner en suspenso las conclusiones de la comunidad científica en lo tocante al cambio climático. El problema, describe McIntyre, es que no hay mejor manera de razonar que la científica aun cuando dentro de, póngase, cincuenta años, se demostrase que sus predicciones eran falsas. Recuérdese que la actitud científica no es un predicado sobre la verdad de nuestras teorías, sino sobre cuál debe ser la manera correcta de comportarse respecto a la evidencia disponible.

Por último, en los capítulos noveno y décimo se trabaja la extensión de la actitud científica hacia otros campos de la ciencia. Disciplinas como la sociología, las ciencias políticas o la antropología, a juicio de McIntyre, adolecen de algunos problemas, como el exceso de teoría, la ausencia de experimentación y replicación, o la renuncia a la causalidad, que pueden amenazar su carácter científico. La solución que considera el autor es clara: "si los científicos sociales estuvieran más preocupados, tanto individualmente como en grupo, en confiar en la evidencia y construir mejores procedimientos con los que capitalizarla, las ciencias sociales estarían en mejor situación (282). El capítulo en cuestión es tentativo y programático, pero a juicio de quien escribe esta nota es sin duda el menos acertado de la obra. La estabilización de la estadística y del paradigma neurobiológico en sociología y psicología respectivamente, han supuesto de facto un acercamiento a la actitud científica sin precedentes que McIntyre parece ignorar. No convence tampoco que el concepto de "evidencia empírica", garante de la actitud científica en ciencias naturales, sea capaz de aglutinar los diferentes tipos de evidencia que manejan, por mencionar alguna disciplina que cita el autor, las ciencias políticas o la economía.

Estas reflexiones me llevan a retomar la anteriormente mencionada idea del naturalismo. Lo cierto es que el enfoque de McIntyre respecto a las ciencias sociales contrasta con la actual tendencia al pluralismo. En los últimos años, se ha venido reivindicando la autonomía de cada disciplina científica -véase el trabajo de la escuela de Stanford 3 ; y no solo de las sociales respecto de las naturales, sino de la biología respecto de la física, de la psicología respecto de la biología, etc. La propuesta de McIntyre supone una vuelta a cierto reduccionismo naturalista: a la idea de que es la evidencia empírica -y no otro tipo de evidencia- el que debe ser respetado y defendido como justificación última del conocimiento. No sé hasta qué punto son compatibles el extender el respeto por la evidencia empírica con el hecho de mantener y preservar la especificad de cada disciplina. Creo que solo tomando la idea de "respeto por la evidencia" en un sentido muy laxo, donde qué cuente como evidencia queda determinado por cada disciplina, es posible tal conciliación. Pero en ese caso la estrategia contra las pseudociencias queda inhabilitada, pues ellas mismas podrían determinar justifi-

3 Entre otros miembros, destacan Ian Hacking, John Dupré o Nancy Cartwright. Véase, Cat (2017) al respecto. 
cadamente su propia evidencia. Parece que el precio a pagar por demarcar la ciencia de la pseudociencia à la McIntyre pasa por rechazar el pluralismo hoy día imperante y por retomar el camino quineano de la naturalización.

Me gustaría sintetizar en este párrafo algunos comentarios críticos que surgen al contemplar las hebras más gruesas de la obra. La actitud científica está plagada de virtudes, pero contiene algún que otro vicio que merece atención. En primer lugar, es improbable que una epistemología de los prejuicios como criterio de demarcación sea exitosa porque uno nunca es consciente de sus propios prejuicios, y cuando lo es, estos dejan de serlo. Así entendida, la ciencia no sería una actividad para el que esté libre de prejuicios, sino para aquel que los consiga ocultar el tiempo suficiente. Además, la posición de McIntyre es naif cuando analiza los procesos introspectivos a través de los que dilucidamos si somos presos del prejuicio -véase la página 274. En segundo lugar, la ciencia "legítima" no está exenta de prejuicios, aunque con asiduidad estos no tienen la forma de ideologías. En muchas ocasiones, los prejuicios de la ciencia han sido más filosóficos que ideológicos; y en este sentido, la filosofía de la ciencia ha brillado en todo su esplendor a la hora de señalarlos. Precisamente la medicina, disciplina sobre la que versa el capítulo sexto, es un caso paradigmático de ciencia que ha avanzado de acuerdo a la actitud científica y a concepciones prejuiciosas sobre la naturaleza del ser humano a partes iguales. Un tercer problema reside en el hecho de que, en última instancia, el "respeto por la evidencia empírica" constituye un estándar que según lo expuesto en el capítulo quinto será tan válido como la comunidad quiera que sea -“la comunidad juzgará qué es y qué no es ciencia” (173). Teniendo esto en mente, uno puede cuestionar qué tiene de especial este estándar frente a cualquier otro igualmente compartido por la comunidad para erigirse como la médula de la demarcación. Un artículo publicado recientemente por Nancy Cartwright y Sindhuja Bhakthavatsalam (2017) sostiene, en esta línea, que la adecuación empírica, estrechamente ligada con la evidencia, es un elemento más a tener en cuenta para la aceptación de una teoría, pero ni siquiera es especialmente relevante a la hora de satisfacer dos tareas que se esperan de toda teoría: proporcionar entendimiento y capacidad de intervención.

Otra debilidad de la tesis de McIntyre consiste en que basar un criterio de demarcación en una determinada actitud -cosa ya de por sí conflictiva- establece un límite temporal a la distinción; concretamente, en el momento en que tal actitud es aceptada por la comunidad científica. Se han escrito mares de tinta sobre el momento histórico en que la evidencia empírica se instituyó como un elemento axial del discurso científico, aunque dejaremos poco margen de error si lo situamos a mediados de siglo XVII en las islas británicas. Distinguir la ciencia de la pseudociencia en base a una actitud respecto a la evidencia empírica implica que ninguna actividad previa al siglo XVII puede ser tildada de científica porque nadie antes reproducía esa actitud. Muchos autores tendrían problemas aceptando esto, otros no. Aquí solo quiero dejar constancia de que la tesis de McIntyre confiere de manera implícita un principio temporal a la ciencia. Por último, en quinto lugar, el mero concepto de "evidencia científica" ha sido suficientemente problematizado por la filosofía de la ciencia -destacando Achinstein (2005) - como para definir la ciencia en base a él. Por mencionar algunos: una misma observación puede servir como evidencia a dos teorías alternativas e incompatibles; fenómenos incompatibles pueden servir como evidencia complementaria a la formulación de una misma teoría; existen corpus de evidencias disponibles que son ignorados durante décadas -e incluso siglos- hasta que son asumidos por otros esquemas conceptuales; en 
ocasiones, es problemático constatar el consenso científico respecto a ciertas evidencias, etc. ¿Qué actitud debe adoptarse en tales casos? ¿qué sucede, para más inri, con aquellas ciencias que operan alejadas de la evidencia, como la matemática? ¿y con aquellas donde la evidencia no es suficiente, como la meteorología? ¿y cuando existen evidencias enfrentadas, cómo se ponderan ceteris paribus? Asumo que estas, precisamente estas, no son cuestiones que preocupan a McIntyre aquí, pero toda concepción que delimite la ciencia a partir de un criterio normativo debería lidiar con los casos límites que pudieran refutarla.

En conclusión, no estoy convencido de que La actitud científica vaya a revitalizar el problema de la demarcación para impulsarlo de nuevo al Olimpo de la discusión filosófica, pues ni se proporcionan soluciones originales a problemas pasados sin resolver, ni se reconfiguran los cimientos del debate ofreciendo vías de escape no concebidas hasta el momento. Con todo, la obra de McIntyre posee profundas y meritorias virtudes que lo convierten en un libro capital de la filosofía de la ciencia contemporánea. En primer lugar, constituye una loable actualización de la filosofía popperiana a la luz del auge de las pseudociencias que pretende poner orden en un escenario donde es frecuente que se desdeñe hasta la más sancionada evidencia. En segundo lugar, esta es una obra que interactúa con su realidad, que dialoga con los conflictos de corte científico de nuestra época, transcendiendo así las engrosadas paredes del mundo académico y mostrando cómo la filosofía de la ciencia es una disciplina comprometida con una mejor erudición de los problemas del presente. Y en tercer y último lugar, La actitud científica promueve una idea tan evidente que solo un filósofo podría empeñarse en criticar: que la ciencia es la mejor manera de comprender e intervenir en el mundo, la que con mayor rigor procede y la que con mayor fiabilidad establece sus resultados, aun cuando, como cualquier práctica humana, no esté exenta de los peligros del fraude, la mentira y el error.

\section{Referencias}

Achinstein, P. (2005), (ed.): Scientific Evidence: Philosophical Theories and Applications, Baltimore: The Johns Hopkins University Press.

Cat, J. (2017), "The Unity of Science”, The Stanford Encyclopedia of Philosophy. (Fall 2017 Edition), Edward N. Zalta (ed.), URL = <https://plato.stanford.edu/archives/fall2017/ entries/scientific-unity/>.

Cartwright, N. \& Bhakthavatsalam, S. (2017), "What's so special about empirical adequacy?", European Journal for the Philosophy of Science, 7(3), pp. 445-465.

Feyerabend, P. (1975), Against Method, London: Verso.

Giere, R. (1999), Science Without Laws, Chicago: The University of Chicago Press.

Laudan, L. (1983), “The demise of the demarcation problem”, en. Cohen, R. S. \& Laudan, L. (eds.): Physics, Philosophy and Psychoanalysis: Essays in Honor of Adolf Grünbaum. D. Reidel, pp. 111-127.

Longino, H. (1990), Science as Social Knowledge, Princeton: Princeton University Press. McIntyre, L. (2020), La actitud científica, Madrid: Cátedra [Trad. de Rodrigo Neira de The Scientific Attitude, Massachusetts: The MIT Press, 2019].

McIntyre, L. (2018), Post-Truth, Massachusetts: The MIT Press. 